\title{
openheart Is improvement in depression in patients attending cardiac rehabilitation with new-onset depressive symptoms determined by patient characteristics?
}

\author{
Serdar Sever (1) , Patrick Doherty, Su Golder, Alexander Stephen Harrison
}

To cite: Sever S, Doherty P, Golder S, et al. Is improvement in depression in patients attending cardiac rehabilitation with new-onset depressive symptoms determined by patient characteristics?. Open Heart 2020;7:e001264.

doi:10.1136/

openhrt-2020-001264

Received 11 February 2020

Revised 24 June 2020

Accepted 13 July 2020
Check for updates

\section{(c) Author(s) (or their} employer(s)) 2020. Re-use permitted under CC BY. Published by BMJ.

Department of Health Sciences, University of York, York, North Yorkshire, UK

\section{Correspondence to} Serdar Sever; ss2284@york. ac.uk

\section{ABSTRACT}

Background Patients with cardiovascular disease (CVD) commonly experience depressive symptoms which is associated with adverse outcome and increased mortality. Examining the baseline characteristics of cardiac rehabilitation (CR) patients that determine Hospital Anxiety and Depression Scale (HADS) depression outcome may facilitate adjustments in CR programme delivery. This study aims to investigate whether comorbidities, demographic and clinical characteristics of patients, with new-onset post-cardiac event depressive symptoms, determine change in their depression following CR.

Methods Analysing the routine practice data of British Heart Foundation National Audit of Cardiac Rehabilitation between April 2012 and March 2018, an observational study was conducted. Patients with new-onset postcardiac event depressive symptoms and no previous documented history of depression constituted the study population.

Results The analyses included $64658 \mathrm{CR}$ patients (66.24 \pm 10.69 years, $75 \%$ male) with new-onset HADS measures, excluding patients with a history of depression. The comorbidities determining reduced likelihood of improvement in depression outcomes after CR were angina, diabetes, stroke, emphysema and chronic back problems. In addition, higher total number of comorbidities, increased weight, a higher HADS anxiety score, smoking at baseline, physical inactivity, presence of heart failure and being single were other significant determinants. However, receiving coronary artery bypass graft treatment was associated with better improvement.

Conclusion The study identified specific baseline comorbid conditions of patients with new-onset depressive symptoms including angina, diabetes, stroke, emphysema and chronic back problems that were determinants of poorer mental health outcomes (HADS) following CR. Higher total number of comorbidities, increased weight, physical inactivity, smoking, presence of heart failure and being single were other determinants of a negative change in depression. These findings could help CR programmes focus on tailoring the CR intervention around comorbidity, physical activity status, weight management and smoking cessation in patients with new-onset depressive symptoms.

\section{Key questions}

What is already known about this subject?

- Patients with cardiovascular disease often experience depressive symptoms that is associated with increased mortality and adverse outcomes. However, there is a lack of studies which investigate baseline characteristics of cardiac rehabilitation (CR) patients that determine depression outcome in patients with new-onset depressive symptoms.

What does this study add?

- The current study is the first to examine the patient characteristics that determine the change in depressive symptoms following CR, specifically in patients with new-onset post-cardiac event depressive symptoms.

How might this impact on clinical practice?

- This paper shows that patients with new-onset depressive symptoms are less likely to improve their depression levels following CR when they have such comorbidities and patient characteristics at baseline: comorbidities of angina, diabetes, stroke, emphysema, chronic back problems, and higher total number of comorbidities, increased weight, physical inactivity, smoking, presence of heart failure and being single according to multivariate analysis results. These findings could enable CR programmes to taiIor the CR intervention around comorbidities, physical activity status, weight management and smoking cessation in this specific patient population.

\section{INTRODUCTION}

The association of depression with increased mortality is widely demonstrated in patients with cardiovascular disease (CVD) in a previous meta-analysis ${ }^{12}$ as well as its association with poor cardiac prognosis. The American Heart Association recently published a scientific statement which recommends elevating depression to the status of a risk factor for mortality and cardiac morbidity in patients with CVD. ${ }^{3}$ Depression is a common condition, one in five of patients with CVD experience depression ${ }^{4}$ and it is one of the 
leading causes of years lived with disability globally. ${ }^{5}$ Depression is associated with loss of productivity and treatment non-compliance in patients with $\mathrm{CVD},{ }^{6}$ along with increased inpatient and outpatient healthcare utilisation costs. ${ }^{7}$

Cardiac rehabilitation (CR) is essential for the comprehensive management of patients with CVD, and is a multicomponent programme that targets secondary prevention and also improvement of patient's psychosocial health. ${ }^{8}$ A recent Cochrane review has demonstrated that CR is effective ${ }^{9}$ and it reduces depressive symptoms. ${ }^{10}$ The assessment of depression is widely recommended by the recent clinical guidelines as it is a well-established risk factor for worse cardiac prognosis and outcomes. ${ }^{3811} 12$

The time onset of depressive symptoms has been the focus point in the recent studies with regard to cardiac morbidity and mortality. ${ }^{13-16}$ Although some studies revealed that patients with history of depression prior to cardiac event experienced increased cardiac morbidity and mortality, ${ }^{13}{ }^{14}$ other studies have found that patients with new-onset depressive symptoms after cardiac event were more likely to experience adverse cardiac events and mortality. ${ }^{1516} \mathrm{~A}$ recent National Audit of Cardiac Rehabilitation (NACR)-based study has contributed to this literature and found that baseline characteristics of patients with history of depression such as higher anxiety, higher total number of comorbidities, smoking, physical inactivity and male gender were determinants of their depression levels following CR. ${ }^{17}$ Moreover, previous studies were unable to be inclusive of different types of comorbidities and their association with depression outcome in CR setting, and were also unable to adjust for heart failure (HF) and cardiac treatments. Therefore, the current study is the first to examine the impact of comorbidities and other patient characteristics on the depression outcome in the context of UK CR programmes.

\section{METHODS}

The strengthening the reporting of observational studies in epidemiology (STROBE) checklist was employed to report this study. ${ }^{18}$

\section{Data collection}

Patient data based within NACR database were extracted and analysed. The primary aim of the NACR is to monitor CR programmes and improve the quality of delivery in UK CR centres and outcomes. To be able to achieve this, CR programmes collect individual patient data under section 251 approval of the NHS Act 2006 which is entered and secured in an online system held by NHS Digital. The identifiable patient data can be collected by the approval that NHS Digital has and these data are then anonymised before being made available for the NACR. Thus, due to this data governance process, there was no need to gain patient consent from each individual. NHS Digital reviews the data governance agreement between NACR and NHS Digital annually. In the present study, no additional NHS ethical approval was required because the data are used in line with NACR purposes and complies with data protection regulations. Currently, the number of CR services entering the data electronically is 229 which is $80 \%$ of all programmes. ${ }^{19}$ The data include patients' initiating event, demographics, risk factors, treatment, medication and outcomes who undergo CR in the UK.

\section{Participants}

The analysis was based and data extracted from the NACR between 1 April 2012 and 31 March 2018. The study population included patients with myocardial infarction (MI) and HF and those who receive treatment of percutaneous coronary intervention (PCI) and coronary artery bypass graft $(\mathrm{CABG})$ as recommended in the clinical guidelines. ${ }^{2021}$ All the eligible patients who did not present with prior history of depression and who had pre-HADS and post-HADS assessments recorded in CR $(n=64658)$ were selected as participants during the study period.

\section{Measures}

Patients who had no reported prior history of depression and had valid pre-CR and post-CR HADS measurements were selected using the NACR data set, and with this approach, eligible patient population was defined for the study sample. History of depression in the NACR data is confirmed by CR practitioners with case note review and by patients if they have ever been diagnosed or treated by a doctor for depression.

\section{Hospital Anxiety and Depression Scale (HADS)}

The HADS is an assessment tool in the form of a selfanswered questionnaire which is employed for screening depressive symptoms in clinical practice. The HADS is one of the psychosocial health measurements that is recommended for assessment before and after CR to provide patients with tailored CR according to their needs. ${ }^{8}$ There are 14 items in HADS, 7 of which cover anxiety symptoms and 7 depressive symptoms. The score of 0 to 3 can be assigned to each item, accordingly a minimum of 0 and maximum of 21 can be received for the separate anxiety and depression scores, and the higher the scores the worse the symptom is. The HADS has been demonstrated to be a reliable and valid measure for assessing anxiety and depression measurements, and is therefore recommended to use with patients with CVD. ${ }^{22-24}$ In the current study, the analysis and the clinical cut-off point of 8 was used to categorise patients into patients with absence of new-onset depressive symptoms $(<8)$ and presence of newonset depressive symptoms $(\geq 8)$ groups. ${ }^{24}$ The reason for this is that a systematic review has demonstrated that an optimal balance between sensitivity and specificity for HADS as a screening tool was often achieved at a cut-off score of 8 for both HADS anxiety and HADS depression, considering the specificity and sensitivity for both scales at roughly $0.80 .^{23}$ The analysis used in the present study 
then compared the patients with HADS $<8$ and HADS $\geq 8$ in a subgroup of patients without history of depression. In addition, baseline HADS anxiety scores that are routinely reported as part of HADS were employed to see whether it determines depression outcomes after CR.

\section{Total number of comorbidities and comorbidity types}

Total number of comorbidities is defined as the sum of the number of comorbidities that the patients had including angina, diabetes, stroke, emphysema, chronic back problems and others. Comorbidities are defined as the medical history of conditions in the NACR data which is confirmed by CR providers with case note review and by patients answering the question of whether they have been diagnosed or been treated for the condition.

\section{Other variables}

The patient demographics used in the analysis were age, gender and marital status (single/partnered). The English Index of Multiple Deprivation (IMD), a measure of deprivation used in England, was another patient demographic. Seven domains are applied to construct the IMD measure: employment, health deprivation and disability, income, crime, barriers to housing and services, living environment, education skills and training. ${ }^{25}$ In total, there are 32844 sub-areas ranked from the most to least deprived areas. IMD was used to categorise patients into two quintiles where the first quintile, reported as 'lowest quintile', means the most deprived areas and other quintiles categorised as the less deprived. PreCR, baseline smoking measurements are categorised according to whether the patient was a current smoker or non-smoker. Other included variables were weight $(\mathrm{kg})$, moderate physical activity (150 min a week), presence of heart failure (yes/no) and cardiac treatment (PCI/ $\mathrm{CABG} /$ other/none). All the variables included in this paper have been chosen in line with the literature and baseline clinical assessment variables carried out by CR practitioners. These variables were explained in detail in previous publications. ${ }^{172627}$

\section{Statistical analysis}

The data analyses were performed using the IBM SPSS software statistics V.25. Patients without prior history of depression who have pre-HADS and post-HADS assessments recorded constituted the study population. A p value of $<0.05$ was considered to be statistically significant. Summary statistics were presented as means, SD and percentages. The descriptive statistics were calculated, and the baseline characteristics compared between patients with absence of new-onset depressive symptoms and presence of new-onset depressive symptoms, using t-tests for continuous variables and $\chi^{2}$ tests for categorical variables. Cohen's d effect size was calculated for continuous variables and phi or Cramer's V effect size was reported for categorical variables. A binary logistic regression model was conducted in order to investigate

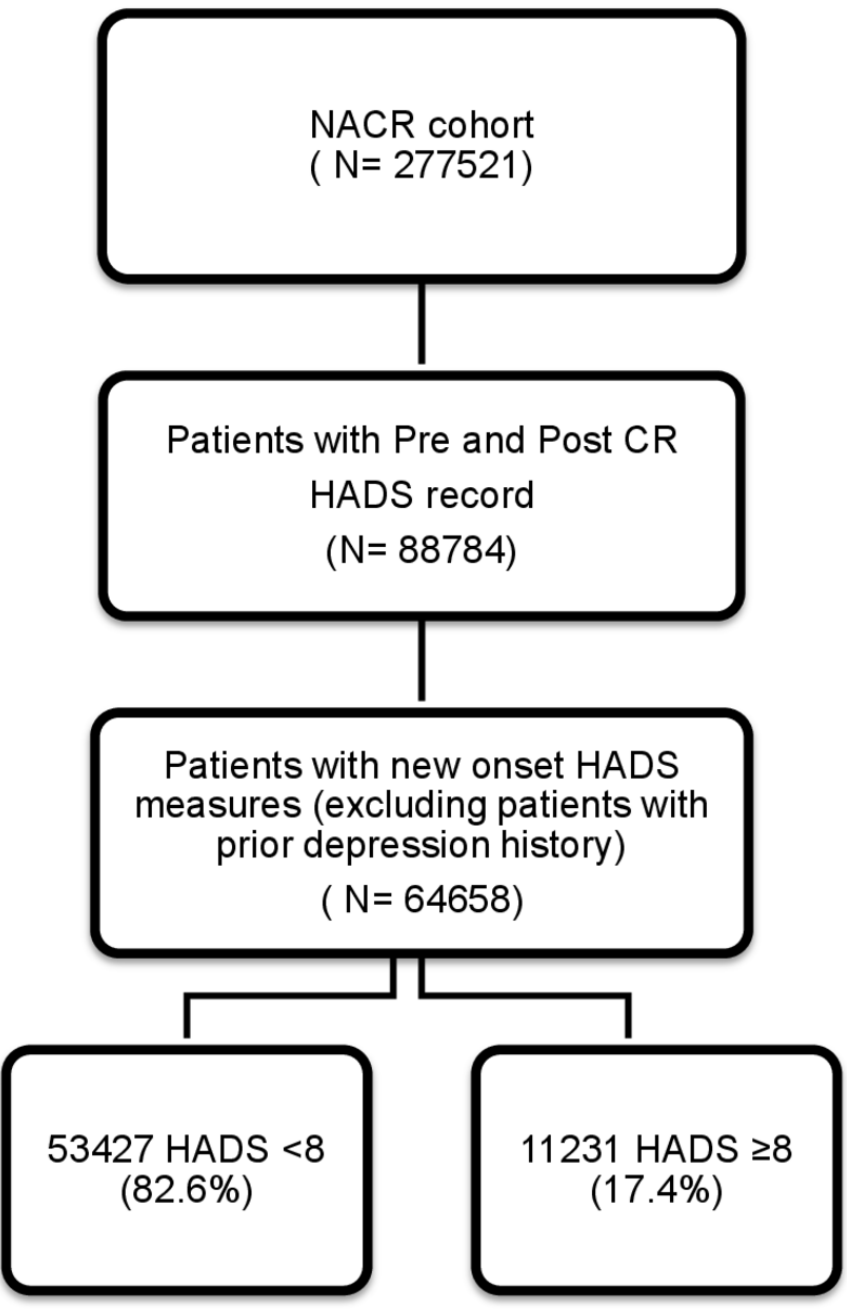

Figure 1 Study flow diagram. CR, cardiac rehabilitation; HADS, Hospital Anxiety and Depression Scale; NACR, National Audit of Cardiac Rehabilitation.

which variables determined improvement in the HADS depressive symptoms following CR.

\section{RESULTS}

A total of 64658 patients without prior history of depression who had completed CR with valid pre-HADS and postHADS assessments constituted the study population. From this population of 64658 participants, $17.4 \%$ presented with new-onset depressive symptoms (HADS $\geq 8$ ) and $82.6 \%$ were, for this study, defined as having an absence of newonset depressive symptoms (HADS $<8$ ). The total population during the study time period and the study sample size are shown in the flow diagram in figure 1. At CR baseline assessment, patients with new-onset depressive symptoms (HADS $\geq 8$ group) differed from the HADS $<8$ group in terms of 11 characteristics in that they were younger, female, single, from areas of higher deprivation, had a higher total number of comorbidities, more weight, higher anxiety scores, physically inactive, more likely to smoke, presence of heart failure and less likely to receive cardiac treatments. The HADS $\geq 8$ group presented with a higher 


\begin{tabular}{|c|c|c|c|c|}
\hline \multirow[b]{2}{*}{ Variables } & \multirow{2}{*}{$\begin{array}{l}\text { HADS }<8 \text { group } \\
(\mathrm{n}=53427)\end{array}$} & \multirow{2}{*}{$\begin{array}{l}\text { HADS } \geq 8 \text { group }(n=11231) \\
\text { Mean } \pm S D\end{array}$} & \multirow[b]{2}{*}{$P$ value } & \multirow[b]{2}{*}{ Effect size } \\
\hline & & & & \\
\hline Age & $66.56 \pm 10.54$ & $64.73 \pm 11.29$ & $<0.001$ & 0.17 \\
\hline Total comorbidities & $2.34 \pm 1.44$ & $2.58 \pm 1.56$ & $<0.001$ & 0.16 \\
\hline Weight & $82.35 \pm 16.46$ & $83.11 \pm 18.40$ & $<0.001$ & 0.05 \\
\hline HADS anxiety score & $4.38 \pm 3.31$ & $9.56 \pm 4.03$ & $<0.001$ & 1.50 \\
\hline Gender female \% & 24.1 & 29.3 & $<0.001$ & 0.05 \\
\hline 150 min physical activity a week (yes) \% & 46.2 & 28.5 & $<0.001$ & 0.14 \\
\hline Smoking (yes) \% & 4.7 & 8.3 & $<0.001$ & 0.06 \\
\hline Single $\%$ & 20.1 & 24.8 & $<0.001$ & 0.04 \\
\hline IMD (most deprived) \% & 9.6 & 15.0 & $<0.001$ & 0.07 \\
\hline Heart failure (yes) \% & 6.4 & 10.1 & $<0.001$ & 0.06 \\
\hline \multicolumn{5}{|l|}{ Comorbidity } \\
\hline Angina \% & 18.4 & 19.5 & 0.008 & 0.01 \\
\hline Diabetes $\%$ & 19.1 & 24.6 & $<0.001$ & 0.05 \\
\hline Stroke \% & 4.2 & 5.9 & $<0.001$ & 0.03 \\
\hline Emphysema \% & 1.7 & 2.6 & $<0.001$ & 0.03 \\
\hline Chronic back problems \% & 11.5 & 14.3 & $<0.001$ & 0.03 \\
\hline \multicolumn{5}{|l|}{ Cardiac treatment } \\
\hline No treatment \% & 8.5 & 11 & $<0.001$ & 0.05 \\
\hline $\mathrm{PCl} \%$ & 50.3 & 44.5 & & \\
\hline CABG \% & 16.6 & 16.2 & & \\
\hline Other treatment \% & 24.6 & 28.3 & & \\
\hline
\end{tabular}

CABG, coronary artery bypass graft; HADS, Hospital Anxiety and Depression Scale; IMD, Index of Multiple Deprivation; PCI, percutaneous coronary intervention.

proportion of comorbidities including angina, diabetes, stroke, emphysema, and chronic back problems compared with those with an HADS $<8$ group. Baseline characteristics of patients according to high and low HADS scores (HADS $<8$, absence of new-onset depressive symptoms; HADS $\geq 8$, presence of new-onset depressive symptoms) are presented in table 1.

A binominal logistic regression was performed to ascertain the impact of the 11 patient characteristics and 5 identified comorbidities on the likelihood that participants' depression symptoms improved between HADS depression symptom group following $\mathrm{CR}$. The logistic regression model was statistically significant, $\chi^{2}(18)=359.814, \mathrm{p}<0.001$. The model correctly classified $63.3 \%$ of the cases. Hosmer and Lemeshow test shows that the model was a good fit $(\mathrm{p}=0.208)$. Of the 16 predictor variables, 13 were statistically significant: weight, HADS anxiety score measurement, physical inactivity, smoking, marital status, total number of comorbidities, presence of heart failure, CABG or other treatments, comorbidities of angina, diabetes, stroke, emphysema and chronic back problems (as shown in table 2).

Patients with higher number of comorbidities had 0.932 times lower odds of residing in low HADS levels after CR.
Increased HADS anxiety score was associated with a $10 \%$ decreased likelihood of improved depressive symptoms after CR. Physical inactivity was also associated with $18 \%$ reduced odds of moving to absence of HADS depressive symptoms category. Smoking was associated with 25\% decreased likelihood of improvement in HADS range after CR. Increased weight and being single were also negative determinants of change in depression. Patients who have the comorbidity of emphysema had 0.665 times lower odds of having improved depressive symptoms which is followed by having the following conditions: comorbidity stroke, comorbidity angina, comorbidity chronic back problems and comorbidity diabetes $(0.718,0.778,0.812$ and 0.830 times, respectively). Patients who had heart failure were $25 \%$ less likely to improve their depressive symptoms following CR. In addition, patients who receive CABG and other treatments were $43 \%$ and $36 \%$ increased odds of having improved depressive symptoms.

\section{DISCUSSION}

Previously, the association of depressive symptoms with poor cardiac prognosis, cardiac mortality and overall mortality is well established in the literature. Yet, there 
Table 2 Coefficients of the model determining change in depression whether a patient has improved depressive symptoms after CR

\begin{tabular}{|c|c|c|c|c|c|c|}
\hline Variable & B & SE & $P$ value & OR & Lower $95 \% \mathrm{Cl}$ & Upper $95 \%$ Cl \\
\hline Age & -0.005 & 0.003 & 0.138 & 0.995 & 0.990 & 1.001 \\
\hline Total no of comorbidities & -0.071 & 0.024 & 0.004 & 0.932 & 0.888 & 0.977 \\
\hline Weight & -0.008 & 0.002 & $<0.001$ & 0.992 & 0.989 & 0.996 \\
\hline HADS anxiety score & -0.105 & 0.008 & $<0.001$ & 0.900 & 0.885 & 0.915 \\
\hline 150 min a week physical activity (no) & -0.196 & 0.067 & 0.003 & 0.822 & 0.721 & 0.937 \\
\hline Smoking (yes) & -0.281 & 0.118 & 0.018 & 0.755 & 0.599 & 0.952 \\
\hline Gender (male) & -0.119 & 0.075 & 0.112 & 0.888 & 0.767 & 1.028 \\
\hline Marital status (single) & -0.273 & 0.073 & $<0.001$ & 0.761 & 0.660 & 0.877 \\
\hline IMD (most deprived) & -0.015 & 0.088 & 0.864 & 0.985 & 0.829 & 1.171 \\
\hline Angina (yes) & -0.251 & 0.083 & 0.003 & 0.778 & 0.661 & 0.916 \\
\hline Diabetes (yes) & -0.186 & 0.077 & 0.016 & 0.830 & 0.714 & 0.965 \\
\hline Stroke (yes) & -0.332 & 0.136 & 0.015 & 0.718 & 0.550 & 0.937 \\
\hline Emphysema (yes) & -0.407 & 0.207 & 0.049 & 0.665 & 0.443 & 0.999 \\
\hline Chronic back problems (yes) & -0.208 & 0.095 & 0.028 & 0.812 & 0.674 & 0.977 \\
\hline Heart failure (yes) & -0.289 & 0.105 & 0.006 & 0.749 & 0.610 & 0.919 \\
\hline \multicolumn{7}{|c|}{ Cardiac treatment (reference: no treatment) } \\
\hline $\mathrm{PCl}$ & 0.078 & 0.116 & 0.499 & 1.082 & 0.862 & 1.357 \\
\hline $\mathrm{CABG}$ & 0.362 & 0.132 & 0.006 & 1.436 & 1.108 & 1.861 \\
\hline Other treatment & 0.313 & 0.119 & 0.008 & 1.367 & 1.084 & 1.725 \\
\hline Constant & 2.720 & 0.320 & $<0.001$ & - & - & - \\
\hline
\end{tabular}

B, regression coefficient; CABG, coronary artery bypass graft; CR, cardiac rehabilitation; HADS, Hospital Anxiety and Depression Scale; IMD, Index of Multiple Deprivation.

remained a need for thorough investigation of the patient characteristics including demographics, comorbidities and clinical characteristics associated with new-onset depressive symptoms. Thus, the current study examined the characteristics of patients with new-onset depressive symptoms in CR patients to a greater extent. The findings of our study demonstrated that baseline characteristics of patients with new-onset depressive symptoms determine the change in the depression outcome after CR. More specifically, patient characteristics such as having a higher number of comorbidities and the comorbidities of angina, diabetes, stroke, emphysema, chronic back problems, increased weight, higher anxiety scores, physical inactivity, smoking, presence of heart failure, CABG treatment and being single were significant predictors of depression outcomes following CR. However, age, gender and IMD were not able to determine depressive symptoms after CR in patients with new-onset depressive symptoms.

A finding of note was that having a higher total number of comorbidities was a significant determinant of depressive symptoms. A prior study employing RCT data in their analysis was unable to find an association between comorbidities and depressive symptoms, ${ }^{28}$ which perhaps could be explained by the younger population (mean age 59.1 \pm 19.8 ) compared with the current study (66.24 \pm 10.69$)$. Indeed, a recent meta-analysis of CR trials recommends future trials involve patients who are more representative of the broader patients with CVD, including patients with comorbidities. ${ }^{9}$ In addition, patients who present with multiple comorbidities are found to be less likely to be referred to or uptake CR which is a primary challenge for healthcare providers and CR programmes. ${ }^{29}{ }^{30}$ Yet, attending CR enables patients with multiple comorbidities to benefit in terms of improving their functional capacity and psychosocial conditions. ${ }^{30} 31$

Another finding was that the comorbidity of diabetes was associated with $17 \%$ reduced likelihood of improvement in depressive symptoms following CR (OR 0.830 $95 \%$ CI 0.714 to 0.965$)$. Patients with diabetes attending CR had reduced physical fitness and increased cardiac risk factors compared with non-diabetic patients. ${ }^{32}$ Individuals with diabetes who participate or complete CR were also significantly less likely than non-participants or no-completers to experience mortality. ${ }^{33} 34$ When the condition of patients with diabetes was taken into account in terms of having a greater cardiac risk profile and lower programme participation rate, recommendations have been made to $\mathrm{CR}$ programmes to target patients with diabetes and involve them in CR. ${ }^{35-37}$ In addition, considering the prevalence of diabetes has been steadily increasing over time, ${ }^{38}$ the medical management of diabetes may relatively be necessary and perhaps can reduce depressive symptoms. 
The stroke comorbidity was influential on the depression outcome in patients with new-onset depressive symptoms after CR. It was associated with reduced odds of improvement in depressive symptoms following CR (OR $0.718,95 \%$ CI 0.550 to 0.937 ). Previous studies have shown that patients with stroke comorbidity were less likely to be referred to ${ }^{29}$ and uptake CR. ${ }^{30}$ However, the study of Marzolini $e t a l^{99}$ found that patients who had a stroke who undergo CR improve their functional capacity and cardiovascular fitness; for that reason, including patients who had a stroke in CR can be beneficial.

Emphysema is the comorbidity which had the highest impact, among the comorbidities, on the change in depressive symptoms following CR. Having emphysema at baseline was associated with $34 \%$ reduced odds of improvement in the depressive symptoms after CR. Patients with chronic obstructive pulmonary disease (COPD) and CVD experience problems of breathlessness and disability in the context of multi-morbidity, thus, cardiac rehabilitation services are recommended to take positive action by providing tailored intervention options for patients with COPD.$^{40}$ In addition, angina and chronic back problems were other comorbidities that were negative determinants of depression outcomes following CR with OR $0.778,95 \%$ CI 0.661 to 0.916 and OR 0.812 , $95 \%$ CI 0.674 to 0.977 , respectively. Overall, all of the comorbidities that were mentioned were negative determinants of improvement in CR patient's depressive symptoms following CR in patients who present with new-onset depressive symptoms. This is the first study showing the influence of the variety of comorbidities on CR outcomes in patients with new-onset depressive symptoms.

Our finding of clinical relevance was that smoking, physical inactivity and weight were modifiable CVD risk factors having an unfavourable impact on depression outcomes after CR. These factors were negative determinants of improvement in depression in patients with new-onset depressive symptoms following CR (OR $0.755,95 \%$ CI 0.599 to 0.952 ; OR $0.822,95 \%$ CI 0.721 to 0.937 ; OR $0.992,95 \%$ CI 0.989 to 0.996 , respectively). These results were in line with previous systematic reviews conducted in the general population, ${ }^{41-43}$ and other cohort studies that involved patients with CVD. ${ }^{44}{ }^{45}$ However, these studies were unable to include CR patients and specifically patients with new-onset depressive symptoms. The current study, on the other hand, included patients who undergo CR and assessed outcome of change in depressive symptoms following CR. Prior studies have tended to focus on CR utilisation and depression with little in the way of evidence regarding factors determining outcome for patients with new-onset depression.

Another finding of the current study is patients with new-onset depressive symptoms and who have higher anxiety score at baseline were less likely to improve their depression symptoms (OR $0.900,95 \%$ CI 0.885 to 0.915 ). This finding demonstrates that anxiety and depression are interrelated and associated with poor outcomes which was in accordance with a previous study. ${ }^{46}$ In the
USA-based study, 622 patients with HF were included and it was revealed that anxiety, measured by Brief Symptoms Inventory, was associated with depressive symptoms in both men and women. In our study, HADS anxiety score measurement was employed for the assessment instead and still confirmed this association. In addition, the current study is first to show that patients with HF were $25 \%$ less likely to improve their depressive symptoms following CR. This result, perhaps, may explain why a recent CR trial was unable to demonstrate improvement in depressive symptoms in HF population. ${ }^{47}$ Thus, patients with HF may need additional psychosocial support from CR teams which may require further investigation in future studies. Moreover, the cardiac treatment was also adjusted in the multivariate analysis in the current study and it has been found that patients who receive the treatment of CABG were $43 \%$ more likely to have improved depressive symptoms.

In the current study, considering patient demographics, being single was associated with $24 \%$ reduced likelihood of improved depressive symptoms after CR (OR 0.761, $95 \%$ CI 0.660 to 0.877 ). Having partner support may improve patients' coping with their illness and thereby improve their depression symptoms. The association of being single with increased depressive symptoms has also been found in meta-analysis of 24 cross-sectional and 8 longitudinal studies conducted by Yan et al. ${ }^{48}$ This study included 52803 individuals from the general population of people aged $\geq 55$ years. In our study, other patient demographics of age, gender and IMD were unable to significantly predict the depression outcome. In the multivariate analysis, the adjustments were made for other cardiac risk factors and comorbidities which may have an impact on this result. Bachmann et at $t^{9}$ recently found an association between lower neighbourhood socioeconomic context and reduced likelihood of participation in CR. In addition, patients who live in socially deprived areas were more disadvantaged than ones who live in less deprived neighbourhoods in terms of inhabiting poor health behaviours such as smoking and being physically inactive. ${ }^{50}$ In the current study, although the IMD was found to be associated with depressive symptoms at baseline assessment, after adjusting for other covariates it has no longer reached statistical significance.

Finally, the model of logistic regression was chosen to match with other literature in this field; for comparisons to be made, future work may look to invoke a propensitybased linear model of change accounting for included and non-included variables.

\section{Limitations}

In order to examine the determinants of outcome, specifically in patients with new-onset depressive symptoms, patients with prior history of depression have been excluded from our population. However, looking at the characteristics of our sample, it was representative of all available patients during the study time scale $(\mathrm{n}=277521)$, mean age was 66.24 compared with 65.06, 
$25 \%$ female compared with $27 \%$, and other variables did not differ by more than $4 \%$. The sample was nationally representative of patients with new-onset depressive symptoms in the UK, yet, it is important to state that not all CR programmes provide a full record of patients who complete CR and in fact, in NACR data, $36.6 \%$ did not have follow-up assessment which may have an influence on the representativeness of the sample. ${ }^{19}$ A strength of this study was the use of an observational approach by employing analysis of routinely collected clinical data which enabled us to generate real-world understanding. In addition, the data involved more patients with multicomorbidities and higher female percentage than prior RCTs. ${ }^{9}$ However, as this is a registry-based study, it should be noted that observational studies do not allow to draw causal conclusions, only association. The analysis was not able to take account of the treatment with antidepressant medication or diagnosis of depression in the CR period, as this was not recorded in the NACR data set.

\section{CONCLUSION}

The primary objective of this study was to ascertain whether comorbidities, demographic and clinical characteristics of patients with new-onset post-cardiac event depressive symptoms determine improvement in their depression following CR. Baseline characteristics of patients with new-onset depressive symptoms such as comorbid conditions of angina, diabetes, stroke, emphysema and chronic back problems, as well as higher total number of comorbidities, increased weight, physical inactivity, smoking, presence of heart failure and being single were negative determinants of improvement in depression following CR. However, receiving CABG or other treatments were positive determinants of improvement. Our findings could promote CR programmes that focus on tailoring the CR intervention around comorbidity, physical activity status, weight management and smoking cessation in patients with new-onset depressive symptoms.

Acknowledgements The authors would like to acknowledge the support of NACR Team.

Contributors SS and PD contributed to the conception or design of the work. SS, $\mathrm{PD}, \mathrm{SG}$ and $\mathrm{ASH}$ contributed to the acquisition, analysis or interpretation of data for the work. SS, PD, SG and ASH drafted and critically revised the manuscript. All authors gave final approval to be accountable for all aspects of the work, ensuring accuracy and integrity.

Funding This research was carried out by the British Heart Foundation (BHF) Cardiovascular Health Research Group, which is supported by a grant from the BHF (grant reference 040/PSS/17/18/NACR).

Competing interests None declared.

Patient consent for publication Not required.

Provenance and peer review Not commissioned; externally peer reviewed.

Data availability statement The data that support the findings of this study are available from the National Audit of Cardiac Rehabilitation, but restrictions apply for the availability of this data, which were used under licence for the current study, as the data is anonymised with NHS Digital under Section 251 and cannot be shared publicly.

Open access This is an open access article distributed in accordance with the Creative Commons Attribution 4.0 Unported (CC BY 4.0) license, which permits others to copy, redistribute, remix, transform and build upon this work for any purpose, provided the original work is properly cited, a link to the licence is given, and indication of whether changes were made. See: https://creativecommons.org/ licenses/by/4.0\%.

ORCID iD

Serdar Sever http://orcid.org/0000-0001-8627-4237

\section{REFERENCES}

1 Meijer A, Conradi HJ, Bos EH, et al. Adjusted prognostic association of depression following myocardial infarction with mortality and cardiovascular events: individual patient data meta-analysis. $\mathrm{Br} \mathrm{J}$ Psychiatry 2013;203:90-102.

2 Meijer A, Conradi HJ, Bos EH, et al. Prognostic association of depression following myocardial infarction with mortality and cardiovascular events: a meta-analysis of 25 years of research. Gen Hosp Psychiatry 2011;33:203-16.

3 Lichtman JH, Froelicher ES, Blumenthal JA, et al. Depression as a risk factor for poor prognosis among patients with acute coronary syndrome: systematic review and recommendations: a scientific statement from the American Heart Association. Circulation 2014;129:1350-69.

4 Thombs BD, Bass EB, Ford DE, et al. Prevalence of depression in survivors of acute myocardial infarction. $J$ Gen Intern Med 2006;21:30-8.

5 GBD 2016 Disease and Injury Incidence and Prevalence Collaborators. Global, regional, and national incidence, prevalence, and years lived with disability for 328 diseases and injuries for 195 countries, 1990-2016: a systematic analysis for the global burden of disease study 2016. Lancet 2017;390:1211-59.

6 Egede LE. Major depression in individuals with chronic medical disorders: prevalence, correlates and association with health resource utilization, lost productivity and functional disability. Gen Hosp Psychiatry 2007;29:409-16.

7 Sullivan M, Simon G, Spertus J, et al. Depression-related costs in heart failure care. Arch Intern Med 2002;162:1860-6.

8 BACPR. Cardiovascular disease prevention and rehabilitation 2017. Br Assoc Cardiovasc Prev Rehabil 2017:1-26.

9 Anderson L, Thompson D, Oldridge N, et al. Exercise-based rehabilitation for coronary heart disease (review). Cochrane Database Syst Rev 2016:10-13.

10 Zheng X, Zheng Y, Ma J, et al. Effect of exercise-based cardiac rehabilitation on anxiety and depression in patients with myocardial infarction: a systematic review and meta-analysis. Heart Lung 2019;48:1-7.

11 SIGN. Cardiac rehabilitation: a national clinical guideline. Scottish Intercoll Guidel Netw 2017:1-42.

12 Piepoli MF, Hoes AW, Agewall S, et al. European guidelines on cardiovascular disease prevention in clinical practice. Eur Heart $J$ 2016;2016:2315-81.

13 Sundbøll J, Schmidt M, Adelborg K, et al. Impact of pre-admission depression on mortality following myocardial infarction. $\mathrm{Br} \mathrm{J}$ Psychiatry 2017;210:356-61.

14 Stenman M, Holzmann MJ, Sartipy U. Association between preoperative depression and long-term survival following coronary artery bypass surgery - a systematic review and meta-analysis. Int $J$ Cardiol 2016;222:462-6.

15 Dickens C, McGowan L, Percival C, et al. New onset depression following myocardial infarction predicts cardiac mortality. Psychosom Med 2008;70:450-5.

16 de Jonge P, van den Brink RHS, Spijkerman TA, et al. Only incident depressive episodes after myocardial infarction are associated with new cardiovascular events. J Am Coll Cardiol 2006:48:2204-8.

17 Sever S, Harrison AS, Golder S, et al. Determinants of depression in patients with comorbid depression following cardiac rehabilitation. Open Heart 2019;6:1-6.

18 Von EE, Egger M, Altman DG, et al. Strengthening the reporting of observational studies in epidemiology (STROBE) statement: guidelines for reporting observational studies. Br Med J 2007;335:806-8.

19 NACR. The National Audit of Cardiac Rehabilitation quality and outcomes report 2018. Natl Audit Card Rehabil 2018.

20 NICE. Chronic heart failure in adults: diagnosis and management. Natl Clin Guidel Cent 2018:NG 106.

21 NICE. Secondary prevention in primary and secondary care for patients following a myocardial infarction. Natl Clin Guidel Cent 2013. 
22 Wang W, Lopez V, Martin C. Structural ambiguity of the Chinese version of the hospital anxiety and depression scale in patients with coronary heart disease. Health Qual Life Outcomes 2006;4:6-4.

23 Bjelland I, Dahl AA, Haug TT, et al. The validity of the Hospital Anxiety and Depression Scale. J Psychosom Res 2002;52:69-77.

24 Zigmond AS, Snaith RP. The Hospital Anxiety and Depression Scale. Acta Psychiatr Scand 1983;67:361-70.

25 Department for Communities and Local Government. The English Indices of Deprivation 20152015.

26 Sever S, Golder S, Doherty P. Factors associated with acute depressive symptoms in patients with comorbid depression attending cardiac rehabilitation. BMC Cardiovasc Disord 2018;18:1-8.

27 Sever S, Doherty P, Harrison AS, et al. To what extent is multimorbidity associated with new onset depression in patients attending cardiac rehabilitation? BMC Cardiovasc Disord 2019;19:1-9.

28 Vitinius F, Escherich S, Deter H-C, et al. Somatic and sociodemographic predictors of depression outcome among depressed patients with coronary artery disease-a secondary analysis of the SPIRR-CAD study. BMC Psychiatry 2019;19:1-9.

29 Brown TM, Hernandez AF, Bittner V, et al. Predictors of cardiac rehabilitation referral in coronary artery disease patients: findings from the American Heart Association's Get With The Guidelines Program. J Am Coll Cardiol 2009;54:515-21.

30 Suaya JA, Shepard DS, Normand S-LT, et al. Use of cardiac rehabilitation by Medicare beneficiaries after myocardial infarction or coronary bypass surgery. Circulation 2007;116:1653-62.

31 Milani RV, Lavie CJ. Impact of cardiac rehabilitation on depression and its associated mortality. Am J Med 2007;120:799-806.

32 Mourot L, Boussuges A, Maunier S, et al. Cardiovascular rehabilitation in patients with diabetes. J Cardiopulm Rehabil Prev 2010;30:157-64

33 Jiménez-Navarro MF, Lopez-Jimenez F, Pérez-Belmonte LM, et al. Benefits of cardiac rehabilitation on cardiovascular outcomes in patients with diabetes mellitus after percutaneous coronary intervention. J Am Heart Assoc 2017;6:1-8.

34 Armstrong MJ, Sigal RJ, Arena R, et al. Cardiac rehabilitation completion is associated with reduced mortality in patients with diabetes and coronary artery disease. Diabetologia 2015;58:691-8.

35 Sumner J, Grace SL, Doherty P. Predictors of cardiac rehabilitation utilization in England: results from the national audit. $J$ Am Heart Assoc 2016;5:1-8.

36 Lopez-Jimenez F, Kramer VC, Masters B, et al. Recommendations for managing patients with diabetes mellitus in cardiopulmonary rehabilitation. J Cardiopulm Rehabil Prev 2012;32:101-12.
37 Banzer JA, Maguire TE, Kennedy CM, et al. Results of cardiac rehabilitation in patients with diabetes mellitus. Am J Cardiol 2004;93:81-4.

38 Einarson TR, Acs A, Ludwig C, et al. Prevalence of cardiovascular disease in type 2 diabetes: a systematic literature review of scientific evidence from across the world in 2007-2017. Cardiovasc Diabetol 2018;17:1-19.

39 Marzolini S, Tang A, Mcllroy W, et al. Outcomes in people after stroke attending an adapted cardiac rehabilitation exercise program: does time from stroke make a difference? J Stroke Cerebrovasc Dis 2014;23:1648-56.

40 Man WD-C, Chowdhury F, Taylor RS, et al. Building consensus for provision of breathlessness rehabilitation for patients with chronic obstructive pulmonary disease and chronic heart failure. Chron Respir Dis 2016;13:229-39.

41 Schuch FB, Vancampfort D, Firth J, et al. Physical activity and incident depression: a meta-analysis of prospective cohort studies. Am J Psychiatry 2018;175:631-48.

42 Fluharty M, Taylor AE, Grabski M, et al. The association of cigarette smoking with depression and anxiety: a systematic review. Nicotine Tob Res 2017;19:3-13.

43 Luppino FS, de Wit LM, Bouvy PF, et al. Overweight, obesity, and depression. Arch Gen Psychiatry 2010;67:220.

44 Stafford L, Berk M, Jackson HJ. Tobacco smoking predicts depression and poorer quality of life in heart disease. BMC Cardiovasc Disord 2013;13:13.

45 Ye S, Muntner P, Shimbo D, et al. Behavioral mechanisms, elevated depressive symptoms, and the risk for myocardial infarction or death in individuals with coronary heart disease: the REGARDS (reason for geographic and racial differences in stroke) study. J Am Coll Cardiol 2013;61:622-30.

46 Eastwood J-A, Moser DK, Riegel BJ, et al. Commonalities and differences in correlates of depressive symptoms in men and women with heart failure. Eur J Cardiovasc Nurs 2012;11:356-65.

47 Dalal HM, Taylor RS, Jolly K, et al. The effects and costs of homebased rehabilitation for heart failure with reduced ejection fraction: the REACH-HF multicentre randomized controlled trial. Eur J Prev Cardiol 2019;26:262-72.

48 Yan X-Y, Huang S-M, Huang C-Q, et al. Marital status and risk for late life depression: a meta-analysis of the published literature. J Int Med Res 2011;39:1142-54.

49 Bachmann JM, Huang S, Gupta DK, et al. Association of neighborhood socioeconomic context with participation in cardiac rehabilitation. J Am Heart Assoc 2017;6:1-13.

50 Stimpson JP, Ju H, a RM, et al. Neighborhood deprivation and health risk behaviors in NHANES III. Third National Health and Nutrition Examination Survey. Am J Health Behav 2007;31:215-22. 